\title{
Radiation hardening and optical properties of materials based on $\mathrm{SiO}_{2}^{*}$
}

\author{
Vladimir A. Stepanov ${ }^{1,2}$, Pavel V. Demenkov ${ }^{3}$, Olga V. Nikulina ${ }^{4}$ \\ 1 Obninsk Institute for Nuclear Power Engineering, NRNU MEPhI, 1 Studgorodok, 249040, Obninsk, Kaluga Reg., Russia \\ 2 FGBUN Interdepartmental Center of Analytical Research in the Field of Physics, Chemistry and Biology at the Presidium of the Russian \\ Academy of Sciences, 65 Bldg. 6 Profsoyuznaya Str., 117997, Moscow, Russia \\ 3 JSC SSC RF-IPPE n.a. A.I. Leypunsky, 1 Bondarenko Sq., 249033, Obninsk, Kaluga Reg., Russia \\ 4 ORPE Technologiya n.a. A.G. Romashin, 15 Kievskoe Hwy, 249031, Obninsk, Kaluga Reg., Russia \\ Corresponding author: Vladimir A. Stepanov (stepanov@iate.obninsk.ru)
}

Academic editor: Yury Kazansky • Received 6 August 2020 • Accepted 17 March 2021 Published 24 June 2021

Citation: Stepanov VA, Demenkov PV, Nikulina OV (2021) Radiation hardening and optical properties of materials based on $\mathrm{SiO}_{2}$. Nuclear Energy and Technology 7(2): 145-150. https://doi.org/10.3897/nucet.7.69926

\begin{abstract}
Preliminary studies have shown that the optical absorption spectra of radiation-colored glasses correspond to the spectral behavior of the scattering losses of an optically inhomogeneous medium. The reasons for the same optical changes in glasses of different compositions are the radiation-induced electric charge separation in the structurally nano-inhomogeneous glass volume, polarization and formation of nanometer optical inhomogeneities.

The authors of this work prove that the radiation changes in the mechanical and optical properties of silicate glasses are of the same nature. The performed estimates indicate that the electric charge separation in the glasses occurs up to absorbed doses of about $1 \mathrm{MGy}$. The local electric charge separation due to the appearance of Coulomb forces leads to radiation hardening of the glasses. The estimated Coulomb hardening of the quartz glasses was $\sim 10^{7} \mathrm{~Pa}$.

The theoretical results were experimentally confirmed by measuring the mechanical properties of the glasses under high intensity proton irradiation as well as by testing the mechanical strength of a composite material based on quartz glass. Under proton irradiation with a dose rate of $5 \times 10^{3} \mathrm{~Gy} / \mathrm{s}$ (energy of $8 \mathrm{MeV}$ ) up to threshold doses of $\sim(1-5) \times 10^{6} \mathrm{~Gy}$ in the KU-1 quartz glasses, the decrement of acoustic vibrations decreased due to Coulomb hardening. After gamma irradiation with $1.34 \times 10^{5} \mathrm{~Gy}$, the tensile strength of the composite material based on quartz glass increased by up to $20 \mathrm{MPa}$. This value is in the range of estimates of Coulomb hardening of quartz glasses. It is also shown that ionizing radiation does not affect the elastic modulus of materials based on $\mathrm{SiO}_{2}$.
\end{abstract}

\section{Keywords}

Silica glass, gamma irradiation, optical properties, acoustic measurement, radiation hardening

\section{Introduction}

The use of glasses as components in the optical systems of radiation devices has a number of significant limitati- ons associated with the decreasing spectral transparency range under irradiation. For example, the spectral transparency range of quartz glasses as a result of irradiation can narrow from $0.2-4.0 \mu \mathrm{m}$ to $0.3-2 \mu \mathrm{m}$ (Arbuzov 2008).

* Russian text published: Izvestiya vuzov. Yadernaya Energetika (ISSN 0204-3327), 2021, n. 1, pp. 143-153. 
Fluoride glasses are transparent in a wider spectral range, from 0.2 to $7.0 \mu \mathrm{m}$, which after irradiation decreases up to 0.4-6.0 $\mu \mathrm{m}$ (Stepanov et al. 2002). However, for all types of glasses, regardless of their chemical composition, in the short-wavelength part of the spectrum, after irradiation, the fundamental absorption edge is blurred and shifted towards longer wavelengths.

Figures 1, 2 show as an example the optical absorption spectra of quartz and fluoride glasses before and after irradiation with a ${ }^{60} \mathrm{Co}$ gamma source. The KU-1 quartz glasses contain a minimum amount of metallic impurities including $\mathrm{Al}, \mathrm{Fe}, \mathrm{Na}$, i.e., less than $0.001 \%$. In the KI glasses, on the contrary, the content of metallic impurities ( $\mathrm{Zr}, \mathrm{Sr}, \mathrm{As}, \mathrm{Mo}$ and $\mathrm{Ba}$ ) reaches $1 \%$. Therefore, the absorption edge of the KI glasses is shifted to the red region. The fluoride glasses are multicomponent, they consist of 5-7 different fluoride compounds:

- fluorozirconate glass (ZBLAN) -

$52 \% \mathrm{ZrF}_{4} 20 \% \mathrm{BaF}_{2} 4 \% \mathrm{LaF}_{3} 4 \% \mathrm{AlF}_{3} 20 \% \mathrm{NaF}$;

- fluoroaluminate glass (AFG) -

$36 \% \mathrm{AlF}_{3} 12.3 \% \mathrm{BaF}_{2} 12.3 \% \mathrm{SrF}_{2} 12.3 \% \mathrm{CaF}_{2} 12.3 \% \mathrm{M}-$ $\mathrm{gF}_{2} 12.3 \% \mathrm{YF}_{3} 2.5 \% \mathrm{LaF}_{3}$.

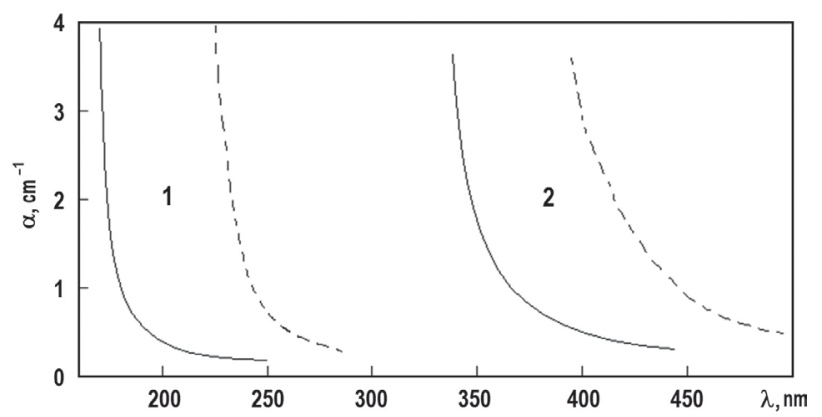

Figure 1. Optical absorption spectra of the silica glasses before (solid lines) and after gamma irradiation (dashed lines): 1 KU-1 (dose $\left.1 \times 10^{8} \mathrm{~Gy}\right) ; 2$ - KI (dose $\left.4 \times 10^{5} \mathrm{~Gy}\right)$.

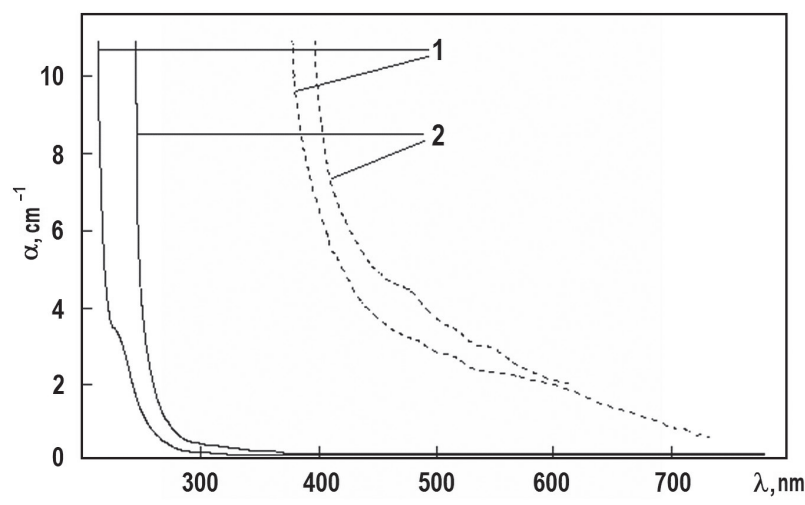

Figure 2. Optical absorption spectra of the fluoride glasses before (solid lines) and after gamma irradiation (dashed lines): 1 - ZBLAN (dose $1.2 \times 10^{6} \mathrm{~Gy}$ ); 2 - AFG (dose $5 \times 10^{4}-1 \times 10^{5} \mathrm{~Gy}$ ).

After irradiation, the fundamental absorption edge is blurred and shifted toward longer wavelengths. Such changes in the optical absorption spectra of the glasses cannot be explained by the appearance, upon irradiation, of structural defects, i.e., centers of optical transitions. In this case, we have to assume that irradiation, regardless of the type of glass, forms a continuous series of color centers with a continuous spectrum of energy levels, at which optical transitions become possible in the entire visible and ultraviolet ranges. The identical nature of the radiation changes in the optical properties of glasses of various compositions is due to the common nature of their nanostructure inherent for all the glasses (Stepanov et al. 2002, Stepanov 2002). In our work, we also show that radiation changes in the mechanical properties of the glasses, along with changes in their optical spectra, occur due to their structural micro- and nano-inhomogeneity.

\section{Structure and optical properties of irradiated glasses}

As early as in 1921, A.A. Lebedev suggested that silica glass has a cryptocrystalline structure, i.e., it consists of a large number of tiny crystallites. According to this hypothesis, the structure of the glasses can be considered as an inhomogeneous medium of microstructural formations, each of which is characterized by an ordered arrangement of atoms. Microinhomogeneity of the glasses was confirmed by various research methods (Malinovsky and Surovtsev 2000). Therefore, most researchers recognize glass as inhomogeneous both in terms of its chemistry and structure. Glasses represent a special disordered state of a solid, in which, however, in nanoregions, the order in the position of atoms is preserved. Such regions (domains), the size of which is determined by the correlation radius of the short-range order of the structure, can vary in the range $d=1-10 \mathrm{~nm}$. Regions of the same size with a disordered structure can be located between these domains (Malinovsky and Noviokov 1992; Malinovsky et al. 2000).

Ionizing radiation leads to the electric charge separation due to the recharging of electron-hole traps and the appearance of optical inhomogeneities in the glass volume due to the polarization of the volume near the charged centers (Stepanov 2002). Radiation current carriers (electrons and holes) migrate to the structural inhomogeneities, to the domain boundaries. This leads to the appearance of separated regions of coherent light scattering of size $d$. An analysis of the absorption spectra made it possible to conclude that the spectral behavior of optical loss in radiation-colored glasses most closely matches the spectral behavior of scattering losses in an optically inhomogeneous medium (Stepanov et al. 2002, Stepanov 2002). Since the wavelength of light in the visible range is $\lambda>30 d$, the optical loss due to scattering and the spectral behavior of the absorption (loss) coefficient can be described using the Rayleigh scattering theory (Fabelinsky 1965):

$$
\alpha=\frac{4 \pi r_{e}^{2}}{3} \cdot \frac{\left(n_{e} d^{3}\right)^{2}}{\left[\left(\lambda / \lambda_{0}\right)^{2}-1\right]^{2}} \cdot n_{d},
$$

where $r_{e}$ is the classical radius of an electron $\left(2.8 \cdot 10^{-15} \mathrm{~m}\right)$; $\lambda_{0}$ is the wavelength corresponding to the forbidden gap; 
$n_{e}$ is the concentration of valence electrons, in order of magnitude atomic concentration; $n_{d}$ is the concentration of domains active in the scattering. If we assume that all the absorbed energy of gamma radiation $E_{g}$ is spent on the creation of charge carriers, then with an increase in the absorbed dose $D$ in the absence of electron-hole annihilation, when all the free electrons are captured by the traps, the concentration of the domains active in scattering increases as

$$
n_{d}=\frac{D \rho}{E_{g}} \cdot \frac{1}{6 d^{2} n_{e}^{2 / 3}},
$$

where $\rho$ is the density of the glass. Substituting (2) into (1), we obtain the dose dependence:

$$
\alpha=\frac{2 \pi r_{e}^{2}}{9} \cdot \frac{D \rho}{E_{g}} \cdot n_{e}^{4 / 3} \cdot \frac{1}{\left[\left(\lambda / \lambda_{0}\right)^{2}-1\right]^{2}} \cdot d^{4} .
$$

The size of the light-scattering domains in accordance with this mechanism of radiation-induced optical losses in the glasses is determined by the short-range order correlation radius, which also corresponds to the size of the smallest structural elements, and the size of the regions of coherent scattering of X-ray radiation. Calculated from the optical spectra of irradiated glasses (Fig. 1) using formula (3), the sizes of coherent light-scattering regions at 300-600 nm: $1.1 \mathrm{~nm}$ for KU-1, $7 \mathrm{~nm}$ for AFG 3.5 , and $5 \mathrm{~nm}$ for ZBLAN, correspond well to the values of the coherent scattering sizes (structural blocks) calculated from the X-ray diffraction spectra: $\mathrm{KU}-1=1.0 \mathrm{~nm}$, $\mathrm{AFG}=3.2 \mathrm{~nm}, \mathrm{ZBLAN}=4.2$. Discrepancies between the optical and X-ray values occur when the glasses contain deep electron trapping centers, for example, impurities of transition metal ions. For example, for the KI glass, the value of $8.1 \mathrm{~nm}$ calculated from the optical spectra significantly exceeds the value of the coherent X-ray scattering region of $1.3 \mathrm{~nm}$. For the gamma-irradiated glasses of various compositions - quartz (KU, KI) and fluoride (fluorozirconate, fluorohafnate, fluoroaluminate) - there is a correlation between the absorption in the short-wavelength part of the spectrum and the initial nanostructure. In accordance with (3), optical loss in irradiated glass strongly depend on the domain size, proportional to $d^{4}$.

It is possible to estimate the saturation dose, at which the entire glass volume is divided into optical scattering regions:

$$
D_{d}=\frac{3 E_{g}}{d \rho} \cdot n_{e}^{2 / 3} \text {. }
$$

This expression for silica glasses gives an estimate of $D_{d} \sim(0.7-2) \times 10^{6} \mathrm{~Gy}$, which is in full agreement with the experimental values of the saturation doses of the radiation color (Arbuzov 2008).

\section{Radiation-induced electric charge separation in inhomogeneous dielectrics}

The studies (Chernov et al. 1998, Plaksin et al. 1999, Plaksin and Stepanov 2001), carried out on ceramic dielectric materials, indicate the effective radiation-induced formation of oppositely electrically charged micro-regions in the volume. It was found that under proton irradiation (energy $=8 \mathrm{MeV}$, dose rate $\left.=1.4 \times 10^{4} \mathrm{~Gy} / \mathrm{s}\right)$, the magnitude of the excess positive charge in $1-10 \mu \mathrm{m}$ grains of $\mathrm{Al}_{2} \mathrm{O}_{3}: \mathrm{Cr}^{3+}$ ceramics $\left(10^{-2}\right.$ mass $\%$ chromium $)$ exceeds $1 \times 10^{-5} \mathrm{C} / \mathrm{cm}^{3}$, and the electric field strength created by this charge is more than $1 \times 10^{4} \mathrm{~V} / \mathrm{cm}$. These values were calculated from the increase in the radioluminescence intensity of the $\mathrm{Cr}^{3+}$ ion band $(690 \mathrm{~nm})$. The mechanism of radioluminescence is due to the capture of electrons by $\mathrm{Cr}^{4+}$ ions and the emission of the resulting $\mathrm{Cr}^{3+}$ ions. In ceramics, electrons sink to the boundaries, which leads to an excess positive charge and an increase in the concentration of $\mathrm{Cr}^{4+}$ ions in the grain. The intensity of this band in $\mathrm{Al}_{2} \mathrm{O}_{3}: \mathrm{Cr}^{3+}$ monocrystals remained constant under the same conditions. The estimation of the electric charge density under proton irradiation at the grain boundaries of the $\mathrm{Al}_{2} \mathrm{O}_{3}: \mathrm{Cr}^{3+}$ ceramics gives $3 \cdot\left(10^{-6}-10^{-5}\right) \mathrm{C} / \mathrm{m}^{2}$. This corresponds to a recharge of up to $10 \%$ of the total amount of chromium ions.

In the quartz glasses, an increase in the radioluminescence intensity with the radiation dose was also associated with radiation-induced charge separation (Demenkov et al. 2000). The local electric charge separation due to the appearance of Coulomb forces leads to the radiation hardening of structurally inhomogeneous dielectric materials. Let us estimate Coulomb glass hardening. We represent the structural elements of the inhomogeneous charge distribution in the volume of the irradiated glass as flat charged capacitors of size $d$ with a charge density on the plates $\rho_{s}$, which is proportional to the density of charged traps for electrons and holes at the domain boundaries. Electric energy of a flat capacitor is

$$
U=\frac{Q^{2}}{2 C},
$$

where $C=\varepsilon \varepsilon_{0} S / d ; S$ is the area of the plates; $d$ is the distance between them. Pressure arises between the plates due to Coulomb attraction

$$
\sigma=-\frac{1}{S} \frac{\partial U}{\partial d}
$$

and, if $Q=\rho_{s} S$, then

$$
\sigma=-\frac{\rho_{s}{ }^{2}}{2 \varepsilon \varepsilon_{0}} .
$$

The minus sign means compression.

In the $\mathrm{Al}_{2} \mathrm{O}_{3}: \mathrm{Cr}^{3+}$ ceramics, under proton ionizing irradiation, up to $1 \times 10^{-1}$ electron-hole traps associated with $\mathrm{Cr}^{3+}$ are charged. If we assume that, in the quartz glasses under irradiation at the domain boundaries, the maximum percentage of charged traps is the same, and their number is on the order of the concentration of Si-O bonds, then we obtain an upper estimate of $1 \times 10^{8} \mathrm{~Pa}$ for the Coulomb hardening. This value, however, is an order of magnitude higher than the yield strength and even the tensile strength (for some glasses). At the domain size $d=1 \mathrm{~nm}$ (e.g., in the KU-1 glasses), up to $30 \mathrm{SiO}_{2}$ formula units are located 
in the surface layer of the same thickness. This gives a lower estimate for the number of charged traps of about $3 \%$ and a lower estimate for hardening up to $1 \times 10^{7} \mathrm{~Pa}$, which is apparently close to real values.

\section{Acoustomechanical properties of silica glasses under proton irradiation}

The electric charge in the glasses is separated, in accordance with (4), up to doses of the order of $1 \mathrm{MGy}$. The dynamics of radiation hardening during irradiation should be the same as the dynamics of optical degradation. To experimentally verify this statement, the mechanical properties of the glasses were measured directly in the process of powerful ionizing proton irradiation using the method of a composite piezoelectric vibrator. The resonance method described in (Nikanorov and Kardashev 1985, Kardashev 2009) was used to measure the acoustomechanical properties of solids (Young's modulus and decrement of acoustic vibrations) directly during irradiation.

The resonance methods are based on the analysis of steady-state forced vibrations of samples in the form of rods or plates. Figure 3 shows a diagram of a composite piezoelectric vibrator, which is an assembly of piezoelectric quartz and a sample of the material under study. The method is based on the direct and reverse piezoelectric effects. Measurements of the resonant frequency and figure of merit of piezoelectric quartz and the piezoelectric quartz-sample system make it possible to calculate the characteristics of the sample. When the vibrator operates under resonance conditions, a standing mechanical wave is formed in it, the location of the nodes and antinodes of which is taken into account when the quartz is fixed and the sample length is selected. The instrumentation and control system allows high accuracy to measure the resonant frequency and the decrement of acoustic vibrations of the composite vibrator. Knowing these parameters, it is possible to calculate the values of Young's modulus $E$ and acoustic vibration decrement $\delta$ of the test sample.

To study changes in the acoustomechanical properties of the samples under powerful irradiation, the composite piezoelectric vibrator was located at the outlet of the ion wire of the EGP-10M light ion accelerator (SSC RF-IP$\mathrm{PE})$ so that a proton beam $1 \mathrm{~cm}$ in diameter fell into the center of the sample (see Fig. 3), where the antinode of the standing ultrasonic wave and the maximum of its amplitude (maximum deformation) are located. The installation made it possible to measure the resonance frequency of the vibrator (Young's modulus) with an error of $0.002 \%$ and the decrement of acoustic vibrations at a frequency of $\sim 100 \mathrm{kHz}$ with an error of $6 \%$ under proton irradiation.

The decrement of acoustic vibrations and Young's modulus of the KU-1 glasses were measured under proton irradiation with energy of $8 \mathrm{MeV}$ and a dose rate of $5 \times 10^{3}$

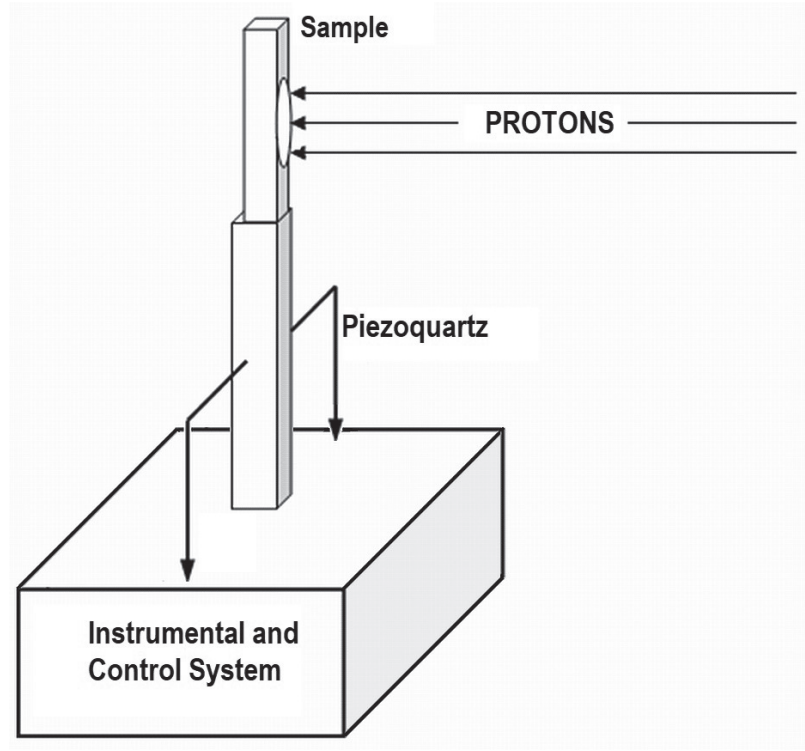

Figure 3. Scheme of measurements of the acoustomechanical properties of the samples under proton irradiation.

Gy/s. The KU-1 glass samples were plates $28.2 \times 3 \times 0.4 \mathrm{~mm}$. The measurements were carried out at a relative deformation of the sample $\sim 1 \times 10^{-6}$. As Figure 4 shows, the decrement of acoustic vibrations first rapidly decreases, reaching in about $200 \mathrm{~s}$ a value of $2.2 \times 10^{-4}$ (dose $\sim 1 \times 10^{6}$ Gy), and then practically does not change throughout the experiment (dose per experiment $\sim 9 \times 10^{6} \mathrm{~Gy}$ ).

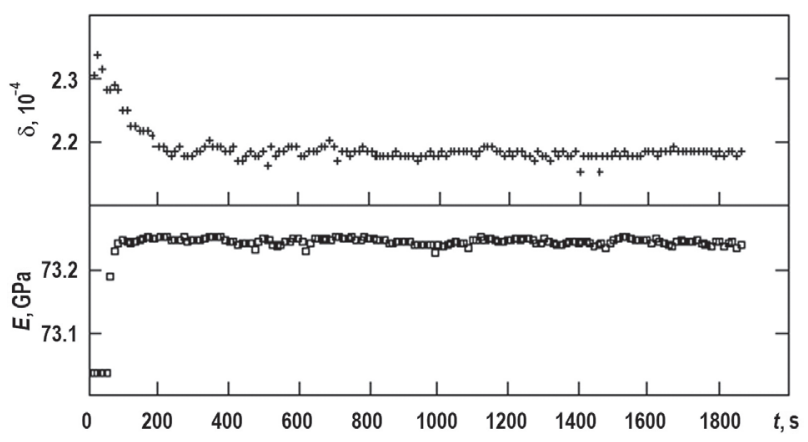

Figure 4. Dependences of Young's modulus $E$ of the KU-1 GLASS and decrement of acoustic vibrations $\delta$ of the composite vibrator under proton irradiation (energy $=8 \mathrm{MeV}$, dose rate $=5 \times 10^{3} \mathrm{~Gy} / \mathrm{s}$ ) at a relative deformation amplitude of $1 \times 10^{-6}$.

The decrement behavior under proton irradiation is associated with the changes in microplasticity and is determined by the formation of stress-strain regions in the glass. A drop in the decrement at the initial stage indicates a decrease in microplasticity due to hardening. The regions with Coulomb hardening arising in the process of ionizing radiation block the motion of defects-carriers of plasticity. A decrease in the decrement of acoustic vibrations is associated with an increase in the number of such regions in the glass. At an irradiation dose of up to $1 \times 10^{6} \mathrm{~Gy}$, in accordance with (4), the hardened micro-regions fill the entire volume, which leads to the completion of the decrement reduction process. 


\section{Mechanical properties of gamma- irradiated silica composites}

An estimate of Coulomb hardening in irradiated silicate glasses gives values of the order of tens of megapascals at absorbed doses close to the saturation dose (4) up to 1 MGy. This hardening leads to an increase in the strength of materials based on $\mathrm{SiO}_{2}$ glasses. OPPE Tekhnologiya, using vacuum and contact molding methods, produces such an inorganic composite material (CAPB brand) for aerospace purposes (Stepanov et al. 2014). As a textured filler, it uses a fabric made of amorphous quartz fiber with an impurity content of no more than $1 \times 10^{-2}$. An inorganic binder based on aluminum phosphates is obtained from aqueous solutions of acidic phosphoric acid salts of aluminum and chromium, to which various dressing agents and micro-grinding powders from electrocorundum and aerosil are added for chemical and mechanical protection of the filler. Such a composite is suitable for operation at temperatures up to $1500{ }^{\circ} \mathrm{C}$.

The strength of the composite is determined by the strength of the quartz filler. An increase in strength from $10 \mathrm{MPa}$ can be detected in mechanical tests of materials, even taking into account the inevitable scatter of the measurement results. To carry out the tests, 30 CAPB samples $(20 \times 10 \times 2) \mathrm{mm}$, were made, half of which were irradiated with a ${ }^{60} \mathrm{Co}$ gamma source. The mechanical tests of the material were carried out in accordance with GOST 4651-82 on an LFM-50 installation; the elastic modulus was calculated in accordance with GOST 9550-81.

The results of mechanical tests are shown in Figs 5, 6 . As in the case of proton irradiation of the quartz glasses,

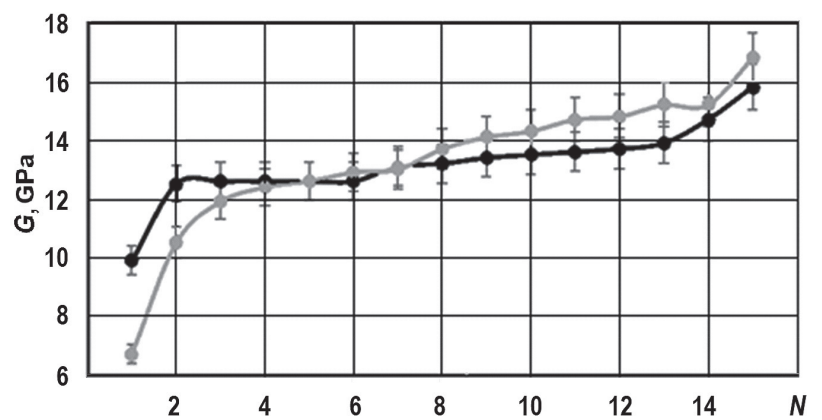

Figure 5. Values of the elastic modulus $G$ during bending of samples $(N)$ of the CAPB samples before (black curve) and after gamma irradiation with a dose of $1.3410^{5} \mathrm{~Gy}$ (gray curve).

\section{References}

- Arbuzov VI (2008) Fundamentals of Radiation Optical Material Science. Saint Petersburg. Saint-Petersburg State University ITMO Publ., 284 pp. [in Russian]

- Chernov VM, Khorasanov GL, Plaksin OA, Stepanov VA, Stepanov PA, Belyakov VA (1998) Electrical and Optical Charac-

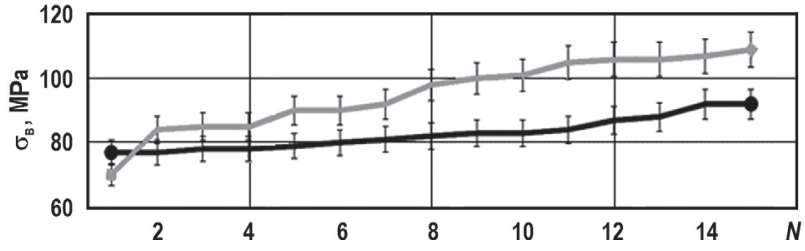

Figure 6. Ultimate strength $\sigma_{\mathrm{B}}$ in bending of the CAPB samples samples before (black curve) and after gamma irradiation with a dose of $1.3410^{5} \mathrm{~Gy}$ (gray curve).

no noticeable changes in the elastic modulus occur after gamma irradiation of the composite based on a fabric of CAPB silica fibers. Figure 5 shows that the values for the irradiated material have both larger and smaller values within the scatter of $2 \mathrm{GPa}$. On the contrary, the ultimate strength of the gamma-irradiated composite exceeds the value of the starting material. In this case, the maximum observed hardening of $20 \mathrm{MPa}$ is in the range of the given estimates of Coulomb hardening of the quartz glass.

\section{Conclusion}

It has been shown that the radiation-induced changes in the mechanical and optical properties of silica glasses are of the same nature and are associated with radiation-induced separation of the electric charge on structural nanoinhomogeneities. The performed estimates indicate that the electric charge is separated in the glasses up to absorbed doses of about 1 MGy. The local electric charge separation leads to radiation hardening of glasses due to the appearance of Coulomb forces. The estimated Coulomb hardening of the quartz glasses was $\sim 1 \times 10^{7} \mathrm{~Pa}$.

The estimates were experimentally confirmed by measuring the mechanical properties of glasses under high intensity proton irradiation as well as by testing the mechanical strength of a composite material based on quartz glass. During proton irradiation with an energy of $8 \mathrm{MeV}$ and a dose rate of $5 \times 10^{3} \mathrm{~Gy} / \mathrm{s}$, in the KU-1 quartz glasses, the decrement of acoustic vibrations (hardening) decreased up to doses of $\sim(1-5) \times 10^{6} \mathrm{~Gy}$. After gamma irradiation of $1.34 \cdot 10^{5} \mathrm{~Gy}$, the tensile strength of the CAPB composite based on a fabric of silica fibers increased by up to $20 \mathrm{MPa}$, and this is in the range of calculations of Coulomb hardening of the quartz glass. It is also shown that ionizing radiation does not affect the elastic modulus of materials based on $\mathrm{SiO}_{2}$. teristics of Dielectrics for Fusion Use under Irradiation. Journal of Nuclear Materials, 253: 175. https://doi.org/10.1016/S00223115(97)00311-5

- Demenkov PV, Plaksin OA, Stepanov VA, Stepanov PA, Chernov VM (2000) Luminescence Kinetics in Proton-Irradiated Quartz 
Glasses. Technical Physics Letters , 26 (6): 475-477. https://doi. org $/ 10.1134 / 1.1262882$

- Fabelinsky IL (1965) Molecular Light Scattering. Moscow. Vysshaya Shkola Publ., 512 pp. [in Russian]

- Kardashev BK (2009) Internal friction and physicomechanical properties of solids. Crystallography Reports, 54 (6): 1021-1032. https:// doi.org/10.1134/S1063774509060170

- Malinovsky VK, Novikov VN, Surovtsev NV, Shebanin AP (2000) Investigation of amorphous states of $\mathrm{SiO}_{2}$ by Raman scattering spectroscopy. Physics of the Solid State, 42: 65-71. https://doi. org $/ 10.1134 / 1.1131169$

- Malinovsky VK, Noviokov VN (1992) The Nature of the Glass Transition and the Excess Low-Energy Density of Vibrational States in Glasses. J. Phys.: Condens. Matter, 4: L139. https://doi. org/10.1088/0953-8984/4/9/003

- Malinovsky VK, Surovtsev NV (2000) Non-Homogeneity on the Nanometer Scale as a Universal Property of Glasses. Fizika i Khimiya Stekla, 26 (3): 315-321. [in Russian]

- Nikanorov SP, Kardashev BK (1985) Elasticity and Dislocation Inelasticity of Crystals. Moscow. Nauka Publ., 256 pp. [in Russian]
- Plaksin OA, Stepanov VA (2001) Radiation-Induced Electrical and Optical Processes in the Materials Based on $\mathrm{Al}_{2} \mathrm{O}_{3}$. Optics and Spectroscopy, 90 (4): 542-551. Translated from Optika i Spektroskopiya, 90 (4): 612-621. https://doi.org/10.1134/1.1366748

- Plaksin OA, Chernov VM, Stepanov PA, Stepanov VA (1999) Radiation-Induced Electrical and Optical Processes in Materials Based on $\mathrm{Al}_{2} \mathrm{O}_{3}$. Journal of Nuclear Materials, 271-272: 496. https://doi. org/10.1016/S0022-3115(98)00807-1

- Stepanov PA, Atroshchenko IG, Starodubtseva NI, Shutkina OV, Melnikov DA (2014) Development of High-Temperature Composite Materials of Heat Protective and Radio Engineering Purposes. Perspektivnye Materialy, 10: 17-21. [in Russian]

- Stepanov VA (2002) Radiation-Induced Changes in Optical Properties of Glasses. Proc. of the $7^{\text {th }}$ Int. Conf. On Engineering Problems of Thermonuclear Reactors. St.-Petersburg, October 28-31: 195. [in Russian]

- Stepanov VA, Baskov PB, Chernov VM, Fedorov VD, Khorozova OD, Kurdyavko PV, Sakharov VV, Stepanov PA (2002) Fluoride Glasses as Materials for Radiation Optics. Proc. of the $13^{\text {th }}$ Int. Symposium on Non-Oxide Glasses and New Optical Glasses, September 9-13. Pardubice, Czech Republic, Part II: 674-677. 